1 Hacettepe Journal of Mathematics and Statistics

$\bigcap$ Volume 48 (1) (2019), 255-273

\title{
Equivariant estimation of quantile vector of two normal populations with a common mean
}

\author{
Manas Ranjan Tripathy ${ }^{* \dagger}$, Adarsha Kumar Jena ${ }^{\ddagger}$ and Somesh Kumar ${ }^{\S}$
}

\begin{abstract}
The problem of estimating quantile vector $\underset{\sim}{\theta}=\left(\theta_{1}, \theta_{2}\right)$ of two normal populations, under the assumption that the means $\left(\mu_{i} \mathrm{~s}\right)$ are equal has been considered. Here $\theta_{i}=\mu+\eta \sigma_{i}, i=1,2$, denotes the $p^{\text {th }}$ quantile of the $i^{\text {th }}$ population, where $\eta=\Phi^{-1}(p), 0<p<1$, and $\Phi$ denotes the c.d.f. of a standard normal random variable. The loss function is taken as sum of the quadratic losses. First, a general result has been proved which helps in constructing some improved estimators for the quantile vector $\underset{\sim}{\theta}$. Further, classes of equivariant estimators have been proposed and sufficient conditions for improving estimators in these classes are derived. In the process, two complete class results have been proved. A numerical comparison of these estimators are done and recommendations have been made for the use of these estimators. Finally, we conclude our results with some practical examples.
\end{abstract}

Keywords: Equivariant estimator, Estimation of quantiles, Complete class results, Common mean, Inadmissibility, Relative risk comparison.

2000 AMS Classification: 62C15, 62F10, 62C20

Received : 22.08.2017 Accepted : 28.06.2018 Doi : 10.15672/HJMS.2018.606

*Department of Mathematics, National Institute of Technology Rourkela, Rourkela-769 008, India, Email: manas@nitrkl.ac.in

${ }^{\dagger}$ Corresponding Author.

$\ddagger$ Department of Mathematics, National Institute of Technology Rourkela, Rourkela-769 008, India, Email: jadarsha@gmail.com

$\S$ Department of Mathematics, Indian Institute of Technology Kharagpur, Kharagpur-721 302, India, Email: smsh@iitkgp.ac.in 


\section{Introduction}

Let $\underset{\sim}{X}=\left(X_{1}, X_{2}, \ldots, X_{m}\right)$ and $\underset{\sim}{Y}=\left(Y_{1}, Y_{2}, \ldots, Y_{n}\right)$ be independent random samples drawn from two normal populations $N\left(\mu, \sigma_{1}^{2}\right)$ and $N\left(\mu, \sigma_{2}^{2}\right)$ respectively. Here the common mean $\mu$, and the variances $\sigma_{1}^{2}, \sigma_{2}^{2}$ are unknown. The $p^{\text {th }}$ quantile of the first and second populations are $\theta_{1}=\mu+\eta \sigma_{1}$ and $\theta_{2}=\mu+\eta \sigma_{2}$ respectively where $\eta=\Phi^{-1}(p)$; $0<p<1$. Here $\Phi($.$) denotes the cumulative distribution function of a standard normal$ random variable. The problem is to estimate the quantile vector $\underset{\sim}{\theta}=\left(\theta_{1}, \theta_{2}\right)$ with respect to the sum of the quadratic losses given by,

$$
L(\underset{d}{d}, \underset{\sim}{\theta})=\sum_{i=1}^{2}\left(\frac{d_{i}-\theta_{i}}{\sigma_{i}}\right)^{2},
$$

where $\underset{\sim}{d}=\left(d_{1}, d_{2}\right)$ is an estimator of $\underset{\sim}{\theta}=\left(\theta_{1}, \theta_{2}\right)$.

The problem of estimation of quantiles has attracted several researchers in the recent past due to its real life applications. For example, quantiles of exponential populations are widely used in the study of reliability, life testing, survival analysis and some related areas. We refer to Keating and Tripathi [7] and Saleh [16] for some applications of exponential quantiles.

We note that, in the literature most of the results on quantile estimation are for a single parameter, $\theta=\mu+\eta \sigma$, whereas the current work is for simultaneous estimation of a vector $\underset{\sim}{\theta}=\left(\theta_{1}, \theta_{2}\right)$ of two quantiles. Probably, Zidek [21] was the first to consider the estimation of quantile of normal population with respect to a quadratic loss function. Zidek [21, 22] proved that the best affine equivariant estimator of the quantile $\theta=\mu+\eta \sigma$ is inadmissible if $|\eta|$ is chosen very large. Rukhin [14] derived a class of minimax estimators for quantile $\theta$, each of which improves upon the best equivariant estimator. For some decision theoretic results on estimation of quantiles of an exponential population one may refer to Rukhin [15] and the references therein.

Some study also has been done in estimating the quantile $\theta_{1}$, when two or more populations are available from normal populations. Kumar and Tripathy [9] considered the estimation of $\theta_{1}=\mu+\eta \sigma_{1}$ under a quadratic loss function using a decision theoretic approach. Exploiting the information available for the common mean, they could obtain improved estimators for quantiles $\theta_{1}$. They also derived some inadmissibility conditions for estimators belonging to equivariant classes. A similar type of results have been obtained by Sharma and Kumar [17] in the case of exponential populations while estimating the quantile $\theta_{1}$ of the first population.

The problem under consideration has its importance in the sense that it uses the information available for estimating a common mean. The problem of estimating the common mean of normal populations is an age old problem and has its origin in the study of recovery of inter-block information in balance incomplete block designs. In the literature, this problem is also referred as Meta-Analysis, where samples (data) from multiple sources are combined with a common objective. One may refer to Vazquez et al. [20] for application of Meta-Analysis in clinical trials. For a detailed review on inference on common mean of two or more normal populations one may refer to Moore and Krishnamoorthy [11], Lin and Lee [10], Chang and Pal [5], Tripathy and Kumar [18] and the references therein.

It should be noted that, the underlying model has been considered previously by Kumar and Tripathy [9], and estimated the first component $\theta_{1}$. We in this paper, consider the simultaneous estimation of quantiles, that is, the vector $\underset{\sim}{\theta}=\left(\theta_{1}, \theta_{2}\right)$, which is important from theoretical as well as application point of view. For some results on simultaneous estimation of location and scale parameters with application we refer to Bai and Durairajan [2], Alexander and Chandrasekar [1] and Tsukuma [19]. The rest 
of our work is organized as follows. In Section 2, we derive a basic result which helps in constructing improved estimators for quantile vector $\underset{\sim}{\theta}$. In Section 3 , we derive affine and location equivariant estimators. Sufficient conditions for improving estimators in the class have been obtained. In the process, two complete class results proved. An extensive simulation study has been done in order to numerically compare the relative risk performances of various proposed estimators in Section 4. We conclude with some practical examples in Section 5.

\section{A General Result and Some Improved Estimators}

In this section we discuss the model and prove a general result which will be handy in constructing some good estimators for the quantile vector $\underset{\sim}{\theta}=\left(\theta_{1}, \theta_{2}\right)$.

Suppose $\underset{\sim}{X}=\left(X_{1}, X_{2}, \ldots, X_{m}\right)$ and $\underset{\sim}{Y}=\left(Y_{1}, Y_{2}, \ldots, Y_{n}\right)$ be independent random samples taken from two normal populations $N\left(\mu, \sigma_{1}^{2}\right)$ and $N\left(\mu, \sigma_{2}^{2}\right)$ respectively. Here the parameters $\mu, \sigma_{1}^{2}$ and $\sigma_{2}^{2}$ are unknown. Our aim is to estimate the vector $\underset{\sim}{\theta}=\left(\theta_{1}, \theta_{2}\right)$, where $\theta_{i}=\mu+\eta \sigma_{i},(\eta \neq 0$ and $i=1,2)$ with respect to the loss function (1.1). Obviously, $\theta_{i}$ is the $p^{t h}$ quantile of the $i^{t h}$ population that is, $\eta=\Phi^{-1}(p),(0<p<1)$ where $\Phi($.$) is$ the cumulative distributive function of a standard normal random variable. A minimal sufficient statistic for this problem is $\left(\bar{X}, \bar{Y}, S_{1}^{2}, S_{2}^{2}\right)$ where

$$
\bar{X}=\frac{1}{m} \sum_{i=1}^{m} X_{i}, \bar{Y}=\frac{1}{n} \sum_{j=1}^{n} Y_{j}, S_{1}^{2}=\sum_{i=1}^{m}\left(X_{i}-\bar{X}\right)^{2} \text { and } S_{2}^{2}=\sum_{j=1}^{n}\left(Y_{j}-\bar{Y}\right)^{2} .
$$

It is well known that the maximum likelihood estimator (MLE) for $\mu$, is not obtainable in a closed form (see Pal et al. [12]). Also the minimal sufficient statistics for this problem are not complete, hence the usual approaches to find uniformly minimum variance unbiased estimator (UMVUE) for individual quantile do not work as ancillary statistics may carry relevant information for the parameter of interest. Therefore, it is not known if a UMVUE exists or not, and it is difficult to find even if one exists. Further, it is known that when we have only one population (say $\underset{\sim}{X}$ ) the best affine equivariant estimator for estimating quantile $\theta_{1}=\mu+\eta \sigma_{1}$ is minimax (see Kiefer [8]). When we have both the populations $\underset{\sim}{X}$ and $\underset{\sim}{Y}$ the problem of estimating the first component $\theta_{1}$ has been considered by Kumar and Tripathy [9]. Following their arguments, a natural way to construct improved estimators for $\underset{\sim}{\theta}$ is to combine the improved estimators for the common mean and the improved estimators for the respective standard deviations. Hence we first propose a basic estimator for $\underset{\sim}{\theta}$ as,

$$
\underset{d}{d}=\left(d_{1}, d_{2}\right), \text { where } d_{i}=\bar{X}+c S_{i}, i=1,2 .
$$

Let us define

$$
c_{m+n}=\frac{\eta \sqrt{2}}{m+n-2}\left[\frac{\Gamma\left(\frac{m}{2}\right)}{\Gamma\left(\frac{m-1}{2}\right)}+\frac{\Gamma\left(\frac{n}{2}\right)}{\Gamma\left(\frac{n-1}{2}\right)}\right] .
$$

2.1. Theorem. If we estimate the quantiles $\underset{\sim}{\underset{\gamma}{b}}$ by $\underset{\sim}{d}=\left(\bar{X}+c S_{1}, \bar{X}+c S_{2}\right)$ with respect to the loss function (1.1), then the value of $c$ for which the risk is minimum is found to be $c_{m+n}$.

Let us denote ${\underset{d}{d}}^{X}=\left(\bar{X}+c_{m+n} S_{1}, \bar{X}+c_{m+n} S_{2}\right)$. Next, we give a general result which in parallel to Theorem 2.1 of Kumar and Tripathy [9] that valid for estimating only $\theta_{1}$.

2.2. Theorem. Suppose $\underset{d_{M}}{d}=\left(d_{M}, d_{M}\right)$ be an estimator for $\underset{\sim}{\mu}=(\mu, \mu)$, and $\underset{\sim}{d}=$ $\left(d_{S_{1}}, d_{S_{2}}\right)$ be an estimator for $\underset{\sim}{\sigma}=\left(\sigma_{1}, \sigma_{2}\right)$. Consider $\underline{d}_{Q}=\left(d_{Q 1}, \widetilde{d}_{Q 2}\right)=\underline{\sim}_{M}+\eta \underline{d}_{S}$ as an 
estimator for $\underset{\sim}{\theta}$. Further, assume that given $d_{S_{1}}$, and $d_{S_{2}}, d_{M}$ is conditionally unbiased for $\mu$, that is

$$
E\left(d_{M} \mid d_{S_{1}}\right)=E\left(d_{M} \mid d_{S_{2}}\right)=\mu
$$

then,

$$
\begin{aligned}
E\left(d_{Q 1}-\theta_{1}\right)^{2}+E\left(d_{Q 2}-\theta_{2}\right)^{2}= & 2 E\left(d_{M}-\mu\right)^{2}+\eta^{2}\left\{E\left(d_{S_{1}}-\sigma_{1}\right)^{2}\right. \\
& \left.+E\left(d_{S_{2}}-\sigma_{2}\right)^{2}\right\}
\end{aligned}
$$

Proof. The proof is similar to the arguments used in proving Theorem 2.1 of Kumar and Tripathy [9], hence omitted.

2.3. Remark. It is easy to observe that, condition (2.3) will satisfy if we choose $d_{M}$ to be an unbiased estimator for $\mu$ and both $d_{S_{1}}$ and $d_{S_{2}}$ are independent of $d_{M}$. For example we may take $d_{M}=\bar{X}$ and $d_{S_{1}}=S_{1}, d_{S_{2}}=S_{2}$.

2.4. Remark. As a consequence of Theorem 2.2, to construct a good estimator for $\underset{\sim}{\theta}$, it is sufficient to have a good estimator for $\mu$ and/or a good estimator for $\sigma_{1}$ or/and a good estimator for $\sigma_{2}$.

2.5. Remark. Let $d_{M}=d_{\phi}$, where $d_{\phi}=\phi\left(S_{1}, S_{2}\right) \bar{X}+\left(1-\phi\left(S_{1}, S_{2}\right)\right) \bar{Y}$ be any unbiased estimator for $\mu$, and $d_{S_{1}}=c S_{1} / \eta, d_{S_{2}}=c S_{2} / \eta(\eta \neq 0)$, it is easy to see that, the condition of Theorem 2.2 satisfies and we prove the following result.

2.6. Theorem. Let $d_{\phi}=\phi\left(S_{1}, S_{2}\right) \bar{X}+\left(1-\phi\left(S_{1}, S_{2}\right)\right) \bar{Y}$ be an estimator for the common mean $\mu$. Consider the estimator ${\underset{\sim}{d}}_{\phi}(c)=\left(d_{\phi}+c S_{1}, d_{\phi}+c S_{2}\right)$ for estimating quantile vector $\underset{\sim}{\theta}$. Then $\underset{\sim}{d_{\phi}}(c)$ has smaller risk than $\underset{\sim}{d}$ with respect to the sum of quadratic loss (1.1) if and only if $d_{\phi}$ has smaller risk than $\overline{\bar{X}}$. Further, $d_{\phi}(c)$ has minimum risk with respect to the loss (1.1) when $c=c_{m+n}$.

We note that, the minimizing choice of $c$ is $c_{m+n}$ which is symmetric in both $m$ and $n$. One may construct an estimator for the quantile $\underset{\sim}{\theta}$ using $\bar{Y}$ for the common mean. Let us denote ${\underset{d}{d}}^{*}=\left(\bar{Y}+c S_{1}, \bar{Y}+c S_{2}\right)$. The results of Theorem 2.6 will remain true if we replace $\underset{\sim}{d} \widetilde{\text { by }} d^{*}$. Hence we have the following remark.

2.7. Remark. Let $d_{\phi}=\phi\left(S_{1}, S_{2}\right) \bar{X}+\left(1-\phi\left(S_{1}, S_{2}\right)\right) \bar{Y}$ be an estimator for the common mean $\mu$. Consider the estimator $\underset{\sim}{d}(c)=\left(d_{\phi}+c S_{1}, d_{\phi}+c S_{2}\right)$ for estimating quantile vector $\underset{\sim}{\theta}$. Then ${\underset{\sim}{\phi}}_{\phi}(c)$ has smaller risk than ${\underset{\sim}{d}}^{*}$ with respect to the sum of quadratic loss (1.1) if and only if $d_{\phi}$ has smaller risk than $\bar{Y}$. Further, $\tilde{d}_{\phi}(c)$ has minimum risk with respect to the loss (1.1) when $c=c_{m+n}$. Let us denote ${\underset{d}{Y}}^{Y}=\left(\bar{Y}+c_{m+n} S_{1}, \bar{Y}+c_{m+n} S_{2}\right)$.

2.8. Remark. Following Theorem 2.6 , one can easily construct good estimators for $\underset{\sim}{\theta}$ by replacing $\bar{X}$ in ${\underset{\sim}{d}}^{X}$ or $\bar{Y}$ in ${\underset{d}{d}}^{Y}$ by any improved estimator of the form $d_{\phi}$ for the common mean $\mu$.

Following the above remarks and Theorem 2.2, we propose the following estimators for $\underset{\sim}{\theta}$ which have smaller risk than ${\underset{\sim}{d}}^{X}$ or/and ${\underset{\sim}{d}}^{Y}$ under certain conditions on the sample 
sizes.

$$
\begin{aligned}
& \underline{d}^{G M}=\left(\hat{\mu}_{G M}+c_{m+n} S_{1}, \hat{\mu}_{G M}+c_{m+n} S_{2}\right), \\
& \underline{d}^{G D}=\left(\hat{\mu}_{G D}+c_{m+n} S_{1}, \hat{\mu}_{G D}+c_{m+n} S_{2}\right), \\
& \underline{d}^{K S}=\left(\hat{\mu}_{K S}+c_{m+n} S_{1}, \hat{\mu}_{K S}+c_{m+n} S_{2}\right), \\
& \underline{d}^{C S}=\left(\hat{\mu}_{C S}+c_{m+n} S_{1}, \hat{\mu}_{C S}+c_{m+n} S_{2}\right), \\
& \underline{d}^{M K}=\left(\hat{\mu}_{M K}+c_{m+n} S_{1}, \hat{\mu}_{M K}+c_{m+n} S_{2}\right), \\
& \underline{d}^{T K}=\left(\hat{\mu}_{T K}+c_{m+n} S_{1}, \hat{\mu}_{T K}+c_{m+n} S_{2}\right), \\
& \underline{d}^{B C 1}=\left(\hat{\mu}_{B C 1}+c_{m+n} S_{1}, \hat{\mu}_{B C 1}+c_{m+n} S_{2}\right), \\
& \underline{d}^{B C 2}=\left(\hat{\mu}_{B C 2}+c_{m+n} S_{1}, \hat{\mu}_{B C 2}+c_{m+n} S_{2}\right) .
\end{aligned}
$$

Here we denote $\hat{\mu}_{G M}=\frac{m \bar{X}+n \bar{Y}}{m+n}, \hat{\mu}_{T K}=\frac{\sqrt{m} b_{n-1} S_{2} \bar{X}+\sqrt{n} b_{m-1} S_{1} \bar{Y}}{\sqrt{m} b_{n-1} S_{2}+\sqrt{n} b_{m-1} S_{1}}$, and $\hat{\mu}_{G D}, \hat{\mu}_{K S}, \hat{\mu}_{B C 1}$, $\hat{\mu}_{B C 2}, \hat{\mu}_{C S}, \hat{\mu}_{M K}$, are estimators for the common mean $\mu$, as defined in Tripathy and Kumar [18]. Although the closed form of the MLE of $\mu$ is not available, one can obtain it numerically by solving a system of three equations in three unknowns. Let us denote $\hat{\mu}_{M L}$ as the MLE of the common mean. Using this estimator for the common mean we propose an estimator for the quantile vector $\underset{\sim}{\theta}$ as,

$$
\underline{d}^{M L}=\left(\hat{\mu}_{M L}+c_{m+n} S_{1}, \hat{\mu}_{M L}+c_{m+n} S_{2}\right) .
$$

All these estimators belong to the class ${\underset{\sim}{d}}^{\phi}\left(c_{m+n}\right)$ and will be compared numerically in Section 4.

2.9. Theorem. Let the estimators ${\underset{d}{d}}^{X},{\underset{d}{b}}^{Y},{\underset{\sim}{d}}^{G D},{\underset{\sim}{d}}^{K S},{\underset{d}{d C 1}}^{B C} \stackrel{d}{ }^{B C 2}$, and ${\underset{d}{d}}^{C S}$ as defined above for estimating $\underset{\sim}{\theta}$. The loss function be taken as the sum of the quadratic losses (1.1).

(i) The estimator ${\underset{\sim}{d}}^{G D}$ performs better than both ${\underset{\sim}{d}}^{X}$ and ${\underset{\sim}{d}}^{Y}$ if and only if $m, n \geq 11$.

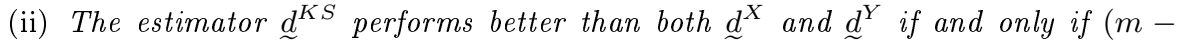
$7)(n-7) \geq 16$.

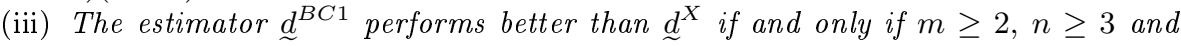
for $0<b_{1}<b_{\max }(m, n)$.

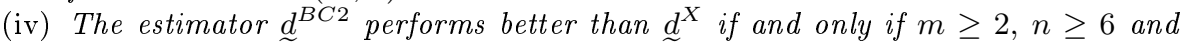
for $0<b_{2}<b_{\max }(m, n-3)$.

(v) The estimator ${\underset{\sim}{d}}^{C S}$ performs better than ${\underset{\sim}{d}}^{X}$ if $m=n \geq 7$.

Here $b_{1}, b_{2}$ and $b_{\max }(m, n)$ are as defined in Kumar and Tripathy [9].

Proof. The proof of (i)-(v) can be done by using Theorem 2.6 and the arguments given in the proof of Theorem 2.4 in Kumar and Tripathy [9].

2.10. Remark. The estimator ${\underset{d}{d K}}^{M K}$ uses the estimator proposed by Moore and Krishnamoorthy [11] that uses the estimates of standard deviation instead of variance. Their estimator does not improve upon $\bar{X}$ uniformly. The estimator ${\underset{\sim}{d K}}^{T K}$ proposed by Tripathy and Kumar [18], also does not improve upon $\bar{X}$ uniformly. As our numerical results shows (in Section 4), these two estimators perform quite well for moderate values of $\sigma_{2} / \sigma_{1}>0$ and also they are good competitor of each other.

\section{Inadmissibility Results for Equivariant Estimators}

In this section, we introduce the concept of invariance to the problem of simultaneous estimation of quantiles of two normal populations and derive classes of affine and location 
equivariant estimators. Further sufficient conditions for improving estimators in these classes have been derived. Consequently some complete class results are also proved.

Consider the group $G_{A}=\left\{g_{a, b}: g_{a, b}(x)=a x+b, a>0, b \in R\right\}$ of affine transformations. Under the transformation, $\bar{X} \rightarrow a \bar{X}+b, \bar{Y} \rightarrow a \bar{Y}+b, S_{i}^{2} \rightarrow a^{2} S_{i}^{2}, \mu \rightarrow a \mu+b$, $\sigma_{i}^{2} \rightarrow a^{2} \sigma_{i}^{2}$, and $\underset{\sim}{\theta} \rightarrow a \underset{\sim}{\theta}+b \underset{\sim}{e}$, where $\underset{\sim}{e}=(1,1)$ and $\underset{\sim}{\theta}=\left(\theta_{1}, \theta_{2}\right), \theta_{i}=\mu+\eta \sigma_{i}, i=1,2$. The problem considered is invariant if we choose the loss function as the sum of affine invariant loss functions (1.1). Based on the sufficient statistics $\left(\bar{X}, \bar{Y}, S_{1}^{2}, S_{2}^{2}\right)$ the form of an affine equivariant estimator for estimating the vector $\underset{\sim}{\theta}$ is obtained as,

$$
\begin{aligned}
\left(d_{1}\left(\bar{X}, \bar{Y}, S_{1}^{2}, S_{2}^{2}\right), d_{2}\left(\bar{X}, \bar{Y}, S_{1}^{2}, S_{2}^{2}\right)\right) & =\left(\bar{X}+S_{1} \Psi_{1}\left(T_{1}, T_{2}\right), \bar{X}+S_{1} \Psi_{2}\left(T_{1}, T_{2}\right)\right) \\
& =\left(d_{\Psi_{1}}, d_{\Psi_{2}}\right) \\
& =\underset{\sim}{d_{\Psi}} \text { say, }
\end{aligned}
$$

where $T_{1}=\frac{\bar{Y}-\bar{X}}{S_{1}}$ and $T_{2}=\frac{S_{2}^{2}}{S_{1}^{2}}$.

Denote $M_{1}=\min \left(t_{1}, 0\right)$, and $M_{2}=\max \left(t_{1}, 0\right)$. Let us define the following functions for any affine equivariant estimator $\underset{\sim}{d_{\Psi}}$.

$$
\begin{gathered}
\stackrel{\Psi}{ }^{0}=\left(\min \left(\max \left(\Psi_{1}, M_{1}\right), M_{2}\right), \min \left(\max \left(\Psi_{2}, M_{1}\right), M_{2}\right)\right) \\
\stackrel{\Psi}{ }^{1}=\left(\max \left\{M_{1}+\eta b_{m+n}, \Psi_{1}\right\}, \max \left\{M_{1}+\eta b_{m+n} \sqrt{t_{2}}, \Psi_{2}\right\}\right), \\
\stackrel{\Psi}{ }^{2}=\left(\min \left\{M_{2}+\eta b_{m+n}, \Psi_{1}\right\}, \min \left\{M_{2}+\eta b_{m+n} \sqrt{t_{2}}, \Psi_{2}\right\}\right) .
\end{gathered}
$$

Next we prove the following inadmissibility result for affine equivariant estimators.

3.1. Theorem. Let $\underset{\sim}{d_{\Psi}}$ be an affine equivariant estimator of the form (3.1) of a quantile vector $\underset{\sim}{\theta}$, and the loss function be the sum of quadratic loss (1.1) or the sum of squared errors. Let the functions ${\underset{\sim}{\Psi}}^{0}, \Psi^{1}$ and $\Psi^{2}$ be defined as in (3.2), (3.3) and (3.4) respectively. Let $\underset{\sim}{\alpha}=\left(\mu, \sigma_{1}^{2}, \sigma_{2}^{2}\right)$.

(i) When $\eta=0$, the estimator ${\underset{\sim}{\Psi}}_{\Psi}$ is improved by ${\underset{\sim}{\Psi^{0}}}_{\mathbb{\sim}^{0}}$ if $P_{\underline{\alpha}}\left(\Psi^{0} \neq \underset{\sim}{\Psi}\right)>0$ for some choices of $\underset{\sim}{\alpha}$.

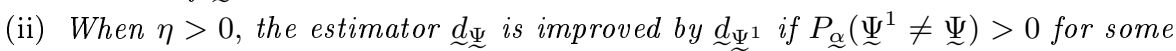
choices of $\underset{\sim}{\alpha}$.

(iii) When $\eta<0$, the estimator $\underset{\sim}{d_{\Psi}}$ is improved by ${\underset{\sim}{\Psi^{2}}}_{\Psi^{2}}$ if $\left(\Psi^{2} \neq \Psi\right)>0$ for some choices of $\underset{\alpha}{\alpha}$.

Proof. To prove this theorem we use a result due to Brewster and Zidek [3]. Consider the conditional risk function of $\underset{\sim}{\underset{\sim}{d}}$ given $\underset{\sim}{T}=\left(T_{1}, T_{2}\right)$ :

$$
\begin{aligned}
& R\left(\left({\underset{\sim}{\Psi}}_{\Psi}, \underset{\sim}{\theta}\right) \mid \underset{\sim}{T}\right)=E\left\{L\left({\underset{\sim}{\Psi}}_{\Psi}, \underset{\sim}{\theta}\right) \mid \underset{\sim}{T}\right\} \\
& =\frac{1}{\sigma_{1}^{2}} E\left\{\left(\bar{X}+S_{1} \Psi_{1}(\underset{\sim}{T})-\mu-\eta \sigma_{1}\right)^{2} \mid \underset{\sim}{T}=\underset{\sim}{t}\right\} \\
& +\frac{1}{\sigma_{2}^{2}} E\left\{\left(\bar{X}+S_{1} \Psi_{2}(\underset{\sim}{T})-\mu-\eta \sigma_{2}\right)^{2} \mid \underset{\sim}{T}=\underset{\sim}{t}\right\} .
\end{aligned}
$$

The above risk function (3.5) is a sum of two convex functions in $\Psi_{1}$ and $\Psi_{2}$, which is a convex function. The minimizing choices of $\Psi_{1}(t)$ and $\Psi_{2}(t)$, are obtained respectively as,

$$
\Psi_{1}(t)=-\frac{E\left\{(\bar{X}-\mu) S_{1} \mid \underset{\sim}{T}\right\}}{E\left(S_{1}^{2} \mid \underset{\sim}{T}\right)}+\eta \sigma_{1} \frac{E\left(S_{1} \mid \underset{\sim}{T}\right)}{E\left(S_{1}^{2} \mid \underset{\sim}{T}\right)}
$$


and

$$
\Psi_{2}(t)=-\frac{E\left\{(\bar{X}-\mu) S_{1} \mid \underset{\sim}{T}\right\}}{E\left(S_{1}^{2} \mid \underset{\sim}{T}\right)}+\eta \sigma_{2} \frac{E\left(S_{1} \mid \underset{\sim}{T}\right)}{E\left(S_{1}^{2} \mid \underset{\sim}{T}\right)} .
$$

Using the conditional expectations derived in Kumar and Tripathy [9], the minimizing choices for $\Psi_{1}(t)$ and $\Psi_{2}(t)$ are simplified and are given by

$$
\Psi_{1}(t, \rho)=\frac{t_{1}}{1+\rho}+\eta b_{m+n} \sqrt{\lambda}
$$

and

$$
\Psi_{2}(t, \rho)=\frac{t_{1}}{1+\rho}+\eta b_{m+n} \sqrt{\frac{n \rho}{m}} \sqrt{\lambda} .
$$

Here $\lambda=\frac{m t_{1}^{2}}{1+\rho}+\frac{m t_{2}}{n \rho}+1, b_{m+n}=\frac{\Gamma\left(\frac{m+n}{2}\right)}{\sqrt{2} \Gamma\left(\frac{m+n+1}{2}\right)}$ and $\rho=\frac{m \sigma_{2}^{2}}{n \sigma_{1}^{2}}$.

In order to prove the theorem, we need to find the infimum and supremum values of $\Psi_{1}(t, \rho)$ and $\Psi_{2}(t, \rho)$ with respect to $\rho>0$, for all values of $\eta$ and $\underset{\sim}{t}$. After analyzing the terms $\Psi_{1}(t, \rho)$ and $\Psi_{2}(t, \rho)$, for separate values of $\eta$, we have the following cases:

(i) When $\eta=0$, and $t_{1} \in \mathbb{R}$,

$$
\begin{gathered}
\inf _{\rho} \Psi_{1}(t, \rho)=M_{1} \text { and } \sup _{\rho} \Psi_{1}(t, \rho)=M_{2} \\
\inf _{\rho} \Psi_{2}(t, \rho)=M_{1} \text { and } \sup _{\rho} \Psi_{2}(t, \rho)=M_{2} .
\end{gathered}
$$

(ii) When $\eta>0$, and $t_{1} \in \mathbb{R}$, we have

$$
\begin{array}{r}
\inf _{\rho} \Psi_{1}(t, \rho) \geq M_{1}+\eta b_{m+n}\left(\text { equality holds if } t_{1}>0\right) \\
\text { and } \sup _{\rho} \Psi_{1}(t, \rho)=+\infty \\
\inf _{\rho} \Psi_{2}(t, \rho) \geq M_{1}+\eta b_{m+n} \sqrt{t_{2}}\left(\text { equality holds if } t_{1}<0\right) \\
\text { and } \sup _{\rho} \Psi_{2}(t, \rho)=+\infty .
\end{array}
$$

(iii) When $\eta<0, t_{1} \in \mathbb{R}$, we have

$$
\begin{array}{r}
\sup _{\rho} \Psi_{1}(t, \rho) \leq M_{2}+\eta b_{m+n}\left(\text { equality holds if } t_{1}<0\right) \\
\text { and } \inf _{\rho} \Psi_{1}(t, \rho)=-\infty \\
\left.\sup _{\rho} \Psi_{2}(t, \rho) \leq M_{2}+\eta b_{m+n} \sqrt{t_{2}} \text { (equality holds if } t_{1}>0\right) \\
\text { and } \inf _{\rho} \Psi_{2}(t, \rho)=-\infty .
\end{array}
$$

Utilizing the expressions (3.8)-(3.10), for $\eta=0, \eta>0$ and $\eta<0$, respectively, for an affine equivariant estimator $\underset{\sim}{d_{\Psi}}=\left(d_{\Psi_{1}}, d_{\Psi_{2}}\right)$, we can easily define the functions ${\underset{\sim}{\Psi}}^{0}, \Psi^{1}$, $\Psi^{2}$ as in (3.2)-(3.4) respectively. An application of orbit-by-orbit improvement technique for improving equivariant estimators of Brewster and Zidek [3], proves the theorem.

3.2. Remark. The above theorem is basically a complete class result. It tells that for an equivariant estimator of the form (3.1),

(i) if $P_{\alpha}\left(\left\{\Psi_{1} \in\left[\min \left(T_{1}, 0\right), \max \left(T_{1}, 0\right)\right]^{c}\right\} \bigcup\left\{\Psi_{2} \in\left[\min \left(T_{1}, 0\right), \max \left(T_{1}, 0\right)\right]^{c}\right\}\right)>0$, then the estimator $\underset{\sim}{d \Psi}$ is improved by $\underset{\Psi_{\Psi^{0}}}{d_{1}}$, when $\eta=0$.

(ii) if $P\left(\left\{\Psi_{1}<\min \left(T_{1}, 0\right)+\eta b_{m+n}\right\} \bigcup\left\{\Psi_{2}<\min \left(T_{1}, 0\right)+\eta b_{m+n} \sqrt{T_{2}}\right\}\right)>0$, then the estimator ${\underset{\sim}{\Psi^{1}}}_{d^{1}}$ will improve upon $\underset{\sim}{d_{\Psi}}$, when $\eta>0$, 
(iii) if $P\left(\left\{\Psi_{1}>\max \left(T_{1}, 0\right)+\eta b_{m+n}\right\} \bigcup\left\{\Psi_{2}>\max \left(T_{1}, 0\right)+\eta b_{m+n} \sqrt{T_{2}}\right\}\right)>0$, then the estimator ${\underset{\sim}{\Psi^{2}}}_{\Psi^{2}}$ will improve upon $\underset{\sim}{d_{\Psi}}$ when $\eta<0$.

Here $[a, b]^{c}$ stands for complement of the interval $[a, b]$ in $\mathbb{R}$.

3.3. Remark. All the estimators discussed in Section 2 (except $\underbrace{M L}$ whose closed form does not exist), belong to the class (3.1). But it has been seen that for none of these estimators, the choices of $\Psi_{1}$ and $\Psi_{2}$ satisfy the above conditions in Remark 3.2. So the estimators considered can not be improved by using Theorem 3.1, but they form a complete class. The result we write as a theorem below.

3.4. Theorem. Let the loss function be (1.1).

(i) The class of estimators $\left\{{\underset{\sim}{\Psi}}_{\Psi}: \Psi_{1} \in\left[\min \left(T_{1}, 0\right), \max \left(T_{1}, 0\right)\right]\right.$ and $\Psi_{2} \in\left[\min \left(T_{1}, 0\right)\right.$, $\left.\left.\max \left(T_{1}, 0\right)\right]\right\}$ is complete for $\eta=0$.

(ii) The class of estimators $\left\{{\underset{\sim}{\Psi}}_{\sim}: \Psi_{1}>\min \left(T_{1}, 0\right)+\eta b_{m+n}\right.$ and $\Psi_{2}>\min \left(T_{1}, 0\right)$ $\left.+\eta b_{m+n} \sqrt{T_{2}}\right\}$ is complete for $\eta>0$.

(ii) The class of estimators $\left\{\underset{\sim}{d \underset{\Psi}{d}:} \Psi_{1}<\max \left(T_{1}, 0\right)+\eta b_{m+n}\right.$ and $\Psi_{2}<\max \left(T_{1}, 0\right)$ $\left.+\eta b_{m+n} \sqrt{T_{2}}\right\}$ is complete for $\eta<0$.

Next, we consider a smaller group of transformations and hence a larger class of estimators for estimating the vector $\underset{\sim}{\theta}$. Consider the group $G_{L}=\left\{g_{c}: g_{c}(x)=c+x, c \in \mathbb{R}\right\}$ of location transformations. Under the transformation, $\bar{X} \rightarrow \bar{X}+c, \bar{Y} \rightarrow \bar{Y}+c, S_{i}^{2} \rightarrow S_{i}^{2}$, $\mu \rightarrow \mu+c, \sigma_{i} \rightarrow \sigma_{i}, \theta_{i}=\mu+\eta \sigma_{i} \rightarrow \theta_{i}+c$ where $i=1,2$.

The estimation problem is invariant if we take the loss function as the sum of squared error losses (1.1), and the form of a location equivariant estimator for estimating the vector $\underset{\sim}{\theta}$ based on the sufficient statistics $\left(\bar{X}, \bar{Y}, S_{1}^{2}, S_{2}^{2}\right)$, is obtained as

$$
{\underset{\sim}{\psi}}_{\mathcal{\psi}}=\left(\bar{X}+\psi_{1}(\underset{\sim}{U}), \bar{X}+\psi_{2}(\underset{\sim}{U})\right) \text {, }
$$

where $\underset{\sim}{U}=\left(T, S_{1}^{2}, S_{2}^{2}\right)$ and $T=\bar{Y}-\bar{X}$.

Let us denote $N_{1}=\min (t, 0)$ and $N_{2}=\max (t, 0)$. For a location equivariant estimator $\underline{\sim}_{\psi}$, define the functions $\underline{\sim}^{0}, \underline{\sim}^{1}$ and $\underline{\sim}^{2}$ as,

$$
\begin{aligned}
\underline{\psi}^{0}(\underline{u})= & \left(\min \left(\max \left(\psi_{1}, N_{1}\right), N_{2}\right), \min \left(\max \left(\psi_{2}, N_{1}\right), N_{2}\right)\right) \\
& \underline{\sim}^{1}(\underline{u})=\left(\max \left\{N_{1}, \psi_{1}\right\}, \max \left\{N_{1}, \psi_{2}\right\}\right), \\
& \underline{\sim}^{2}(\underline{u})=\left(\min \left\{N_{2}, \psi_{1}\right\}, \min \left\{N_{2}, \psi_{2}\right\}\right) .
\end{aligned}
$$

Next, we prove a theorem regarding inadmissibility of location equivariant estimators.

3.5. Theorem. Let $\underset{\sim}{\underset{\sim}{d}}$ be a location equivariant estimator of the quantile $\underset{\sim}{\theta}$ and the loss function be the sum of quadratic losses (1.1) or the sum of squared error. Let the functions ${\underset{\sim}{0}}^{0},{\underset{\sim}{1}}^{1}$ and ${\underset{\sim}{2}}^{2}$ be defined as in (3.12), (3.13) and (3.14) respectively.

(i) When $\eta=0$, the estimator ${\underset{\sim}{\psi}}_{\mathcal{\psi}}$ is improved by ${\underset{\sim}{\psi^{0}}}_{\text {if }}{\underset{\sim}{\alpha}}_{\sim}\left(\underline{\sim}_{0} \neq \underset{\sim}{\psi}\right)>0$ for some choices of $\underset{\alpha}{\alpha}$.

(ii) When $\eta>0$, the estimator ${\underset{\sim}{\psi}}_{\mathcal{\psi}}$ is improved by ${\underset{\sim}{\psi^{1}}}_{\text {if }}{\underset{\sim}{\alpha}}_{\sim}\left(\underline{\sim}^{1} \neq \underset{\sim}{\psi}\right)>0$ for some choices of $\underset{\alpha}{\alpha}$.

(iii) When $\eta<0$, the estimator ${\underset{\sim}{\psi}}_{\mathcal{\psi}}$ is improved by ${\underset{\sim}{\psi^{2}}}$ if $P_{\underset{\alpha}{\alpha}}\left(\underline{\sim}^{2} \neq \underset{\sim}{\psi}\right)>0$ for some choices of $\underset{\sim}{\alpha}$.

Proof. The proof is similar to the arguments used in proving Theorem 3.1. The details of the proof is omitted. 
3.6. Remark. Similar to Theorem 3.1 above Theorem 3.5 is also a complete class result. It tells that for an estimator of the form (3.11),

(i) if $P\left(\left\{\psi_{1} \in[\min (T, 0), \max (T, 0)]^{c}\right\} \bigcup\left\{\psi_{2} \in[\min (T, 0), \max (T, 0)]^{c}\right\}\right)>0$ then the estimator $\underline{\sim}_{\psi}$ is improved by $\underline{\sim}_{\psi^{0}}$, when $\eta=0$,

(ii) if $P\left(\left\{\psi_{1}<\tilde{\min }(T, 0)\right\} \bigcup\left\{\psi_{2}<\tilde{\min }(T, 0)\right\}\right)>0$, then the estimator ${\underset{\sim}{\psi^{1}}}$ will improve upon $\underset{\sim}{d_{\psi}}$, for $\eta>0$, and

(iii) if $P\left(\left\{\psi_{1}>\max (T, 0)\right\} \bigcup\left\{\psi_{2}>\max (T, 0)\right\}\right)>0$, then the estimator ${\underset{\sim}{\psi^{2}}}_{\psi^{2}}$ will improve upon ${\underset{\sim}{\mathcal{L}}}_{\mathcal{L}}$ when $\eta<0$.

3.7. Remark. All the estimators discussed in Section 2 (except ${\underset{d}{d}}^{M L}$ whose closed form does not exist), belong to the class (3.11). But it has also been seen that for none of these estimators the choices of $\psi_{1}$ and $\psi_{2}$ satisfy the above conditions in Remark 3.6. So the estimators considered can not be improved by using Theorem 3.5, but they form a complete class. This we write as a theorem.

3.8. Theorem. Let the loss function be (1.1).

(i) The class of estimators $\left\{\underline{\sim}_{\underset{\psi}{d}}: \psi_{1} \in[\min (T, 0), \max (T, 0)]\right.$ and $\psi_{2} \in[\min (T$, $0), \max (T, 0)]\}$ is complete for $\eta=0$.

(ii) The class of estimators $\left\{\underline{d}_{\mathcal{L}}: \psi_{1}>\min (T, 0)\right.$ and $\left.\psi_{2}>\min (T, 0)\right\}$ is complete for $\eta>0$.

(ii) The class of estimators $\left\{\underline{d}_{\mathcal{\psi}}: \psi_{1}<\max (T, 0)\right.$ and $\left.\psi_{2}<\max (T, 0)\right\}$ is complete for $\eta<0$.

\section{Numerical Comparisons}

In the previous sections we have derived several estimators for the quantile vector

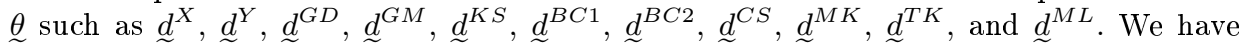
also shown that these well structured estimators, except ${\underset{d}{d L}}^{M L}$, belong to the class (3.1) and (3.11). It seems quite difficult to compare the risk values of all these estimators analytically. But for practical purposes, one needs the estimator to be used. Taking the advantages of computational resources, we in this section compare numerically the simulated risk values of all these estimators which may be handy for practical purposes. For evaluating the risk function, we use the loss function (1.1). For numerical comparison purpose, we have generated 20,000 random samples $\underset{\sim}{X}$ of sizes $m$ and 20,000 random samples $\underset{\sim}{Y}$ of sizes $n$ from normal populations with equal mean and different variances. It can be easily checked that all the risks values are functions of $\tau=\frac{\sigma_{2}}{\sigma_{1}}>0$, for fixed values of $m, n$ and $|\eta|$. The approximate value of $\pi$ is taken to be 3.1416. We have computed the risk values of all the estimators taking various choices of $\tau$ and the sample sizes. However, for illustration purpose we present the risk values for some selected choices of $\tau$ and $m, n$. We also observe that when the values of $\tau$ increase from 0 to $\infty$ the risk values converge for all the estimators except $\underline{d}^{G M}$ and $\underline{d}^{Y}$. As the sample sizes increases the risk values of all the estimators decrease for fixed $|\eta|$. Further, the risk values increase as $\eta$ increases for fixed values of $\tau$ and sample sizes. If we choose the value of $b_{1}$ and $b_{2}$ near

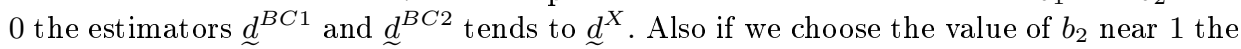

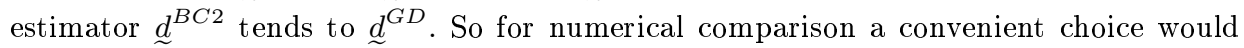
be an intermediate value which we take as $\frac{1}{2} b_{\max }$. The value of $b_{\max }(m, n)$ have been taken from the tabulated values given in Brown and Cohen [4]. We also note that, when the sample sizes are equal the estimator ${\underset{\sim}{d}}^{G D}$ becomes same as ${\underset{\sim}{d}}^{K S}$ and ${\underset{\sim}{d K}}^{M K}$ becomes same as $\underline{d}^{T K}$. When the sample sizes are unequal the estimator $\underline{\sim}^{C S}$ is not defined, so for 
unequal sample sizes we do not include it for numerical comparison purpose. A massive simulation study has been conducted separately for the cases $m=n, m>n$ and $m<n$. The simulated risk values have been plotted against $\tau$ for all the estimators in Figure 1 and Figure 2. In Figure 1 the sample sizes have been taken as equal, whereas in Figure 2, the simulated risk values have been plotted for unequal sample sizes. In Figures 1, and 2 we label $X, Y, G M, G D, K S, B C 1, B C 2, C S, M K, T K$ and $M L$ for the estimators

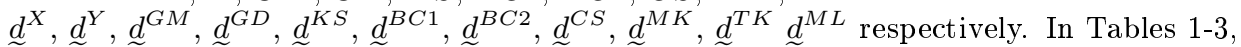
we have presented the simulated values of the percentage of relative risk improvement of all the estimators with respect to ${\underset{\sim}{d}}^{X}$, which are defined as

$$
\begin{aligned}
P R 1 & =\left(1-\frac{\operatorname{Risk}\left(\underline{d}^{Y}\right)}{\operatorname{Risk}\left(\underline{d}^{X}\right)}\right) \times 100, P R 2=\left(1-\frac{\operatorname{Risk}\left(\underline{d}^{G M}\right)}{\operatorname{Risk}\left(\underline{d}^{X}\right)}\right) \times 100, \\
P R 3= & \left(1-\frac{\operatorname{Risk}\left(\underline{d}^{G D}\right)}{\operatorname{Risk}\left(\underline{d}^{X}\right)}\right) \times 100, P R 4=\left(1-\frac{\operatorname{Risk}\left(\underline{d}^{K S}\right)}{\operatorname{Risk}\left(\underline{d}^{X}\right)}\right) \times 100, \\
P R 5= & \left(1-\frac{\operatorname{Risk}\left(\underline{d}^{B C 1}\right)}{\operatorname{Risk}\left(\underline{d}^{X}\right)}\right) \times 100, P R 6=\left(1-\frac{\operatorname{Risk}\left(\underline{d}^{B C 2}\right)}{\operatorname{Risk}\left(\underline{d}^{X}\right)}\right) \times 100, \\
P R 7= & \left(1-\frac{\operatorname{Risk}\left(\underline{d}^{C S}\right)}{\operatorname{Risk}\left(\underline{d}^{X}\right)}\right) \times 100, P R 8=\left(1-\frac{\operatorname{Risk}\left(\underline{d}^{M K}\right)}{\operatorname{Risk}\left(\underline{d}^{X}\right)}\right) \times 100, \\
P R 9= & \left(1-\frac{\operatorname{Risk}\left(\underline{d}^{T K}\right)}{\operatorname{Risk}\left(\underline{d}^{X}\right)}\right) \times 100, P R 10=\left(1-\frac{\operatorname{Risk}\left(\underline{d}^{M L}\right)}{\operatorname{Risk}\left(\underline{d}^{X}\right)}\right) \times 100 .
\end{aligned}
$$

The following observations can be made from the Tables 1-3 and the Figures 1-2 as well as from our simulation study. For illustration purpose, we have presented the risk functions only for the case $\eta=1.960$.

Case 1: $\mathbf{m}=\mathbf{n}$.

(i) Figure 1 represents the risk values of all the estimators for the equal sample sizes and $\eta=1.960$. In Figure 1, (a)-(c) it represents the risk values for sample sizes small to moderate that is $(6,6),(8,8)$ and $(12,12)$ whereas $(d)-(f)$ the sample sizes are taken as moderate to large $(20,20),(30,30)$ and $(40,40)$. It has been noticed

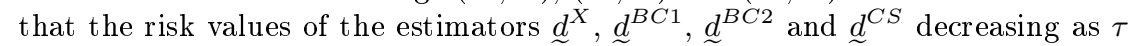
increases from 0 to $\infty$. The estimator ${\underset{\sim}{d}}^{G D}$ first increases and attains maximum value then decreases. The estimators ${\underset{\sim}{d}}^{G M}$, and ${\underset{d}{d K}}^{M K}$ first decrease attains minimum (in the neighborhood of $\tau=1$ ) then increases. The estimator ${\underset{d}{d}}^{Y}$ increases as $\tau$ varies from 0 to $\infty$. It has also been noticed that all the estimators (except $\underline{d}^{G M}$ and $\underline{d}^{Y}$ ) converge to the estimator $\underline{d}^{X}$ which is true as these estimators are consistent.

(ii) The percentage of relative risk performances of all the estimators with respect to $\underline{\sim}^{X}$ decrease as $\tau$ varies from 0 to $\infty$. Let us first consider the case of small sample sizes $(m, n \leq 10)$. For small values of $\tau(\tau<0.25)$ the estimators ${\underset{d}{d}}^{Y}$ and ${\underset{d}{d L}}^{M L}$ has the maximum percentage of relative risk improvement and it is seen near to $98.88 \%$. For moderate values of $\tau(0.75<\tau<2.5)$ the estimators ${\underset{d}{d}}^{G M}$ and ${\underset{d}{d K}}^{M K}$ compete each other however when $\tau=1$, the estimator ${\underset{d}{d}}^{G M}$ has the maximum percentage of relative risk improvement and it is seen near to $15.68 \%$. For large values of $\tau$, the estimator $d^{B C 1}$ has the maximum percentage of relative risk improvement.

Consider the case of moderate sample sizes $(12 \leq m, n \leq 20)$. For small values of $\tau$, the estimator $d^{M L}$ has the best performance and the percentage of relative risk improvement is seen near to $89.78 \%$. For moderate values of $\tau$ 
$(0.75<\tau<2.5)$ the estimators ${\underset{\sim}{d}}^{M K}$ and ${\underset{\sim}{d}}^{G D}$ perform equally well, however for $\tau=1$, the estimator $\tilde{d}^{G M}$ has the maximum percentage of relative risk performances. For large values of $\tau,(\tau>3.5)$ the estimators ${\underset{\sim}{d}}^{B C 1}$ and ${\underset{d}{d L}}^{M L}$ compete with each other.

Consider the case of large sample sizes $(m, n \geq 30)$. For small values of $\tau$ the estimators ${\underset{\sim}{d L}}^{M L}$ and ${\underset{\sim}{d}}^{G D}$ compete with each other and the percentage of relative risk performance has been noticed near to $90.40 \%$. For moderate values of $\tau(0.75<\tau<2.5$,$) the estimators {\underset{d}{d}}^{G D},{\underset{d}{d}}^{M L}$ and ${\underset{\sim}{d K}}^{M K}$ compete with each other, however for $\tau=1$, the estimator $\underline{d}^{G M}$ has the best performance. For large values of $\tau$, the estimators ${\underset{\sim}{d C 1}}^{B C}$ and ${\underset{\sim}{d}}^{B C 2}$ compete with ${\underset{\sim}{d}}^{M L}$.

\section{Case 2: $\mathbf{m}<\mathbf{n}$.}

(i) Figure 2, ((a), (c) and (e)) represents the risk values of all the estimators for $\eta=1.960$ and the sample sizes $(4,10),(12,20)$ and $(30,40)$. The risk values of the estimators $\underline{d}^{X}$, is decreasing as $\tau$ increases. The risk values of $\underline{d}^{G D}, d^{K S}$ increase and attains maximum then decrease as $\tau$ increases. The risk values of all the estimators converge to the risk of $\widetilde{d}^{X}$ except $\widetilde{d}^{Y}$ and $\underline{d}^{G M}$.

(ii) Consider the small sample sizes $(m, n \leq 10)$. For small values of $\tau<0.25$, the estimator ${\underset{d}{d}}^{Y}$ and ${\underset{d}{d L}}^{M L}$ compete with each other and the percentage of relative risk improvement is seen near to $98.88 \%$. For moderate values of $\tau(0.75<$ $\tau<3$, ) the estimators ${\underset{d}{d K}}^{T K}$ and ${\underset{d}{d M}}^{G M}$ compete each other, however for $\tau=$ 1 , the estimator ${\underset{d}{d M}}^{G M}$ has the best performance. For large values of $\tau(\tau>$ 3.0, ) the estimator $d^{B C 1}$ performs the best and the percentage of relative risk performance.

Consider the case of moderate sample sizes $(12 \leq m, n \leq 20)$. For small values of $\tau$ the estimator $\underline{d}^{M L}$ has the maximum percentage of relative risk performance and it is seen near to $98.88 \%$. For moderate values of $\tau(0.75<\tau<3)$ the estimators ${\underset{\sim}{d K}}^{T d^{M K}}$ and $\stackrel{d}{d}^{K S}$ compete each other, however for $\tau=1,{\underset{d}{d M}}^{G M}$ has the best performance. For large values of $\tau(\tau>3)$ the estimator ${\underset{d}{B C 1}}^{B}$ has the maximum percentage of relative risk improvement.

Consider the case of large sample sizes $(m, n \geq 30)$. For small values of

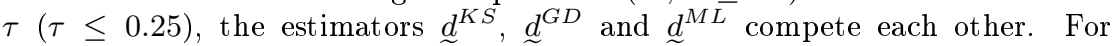
moderate values of $\tau\left(0.25<\tau<3\right.$.) the estimators ${\underset{d}{d}}^{G D}, \stackrel{d}{d}^{K S},{\underset{d}{d K}}^{T K}, \stackrel{d}{ }^{M K}$ and $\widetilde{d}^{M L}$ compete each other. For large values of $\tau$ the estimators ${\underset{d}{d L}}^{M L}$ and ${\underset{d}{d C 1}}^{B C 1}$ compete each other.

\section{Case-3: $m>n$.}

(i) Figure 2, ((b), (d) and (f)) represent the risk values of all the estimators for $\eta=1.960$ and for the sample sizes $(10,4),(20,12)$ and $(40,30)$. The risk values of ${\underset{\sim}{d}}^{X}$ is decreasing as $\tau$ increases. The risk values of ${\underset{\sim}{d}}^{G D},{\underset{\sim}{d}}^{K S}, \sim^{B C 1}$ and ${\underset{d}{d}}^{B C 2}$ decrease as $\tau$ increases. The risk values of estimators $\widetilde{\sim}^{G M}$, and $\widetilde{d}^{Y}$ first decrease attains minimum then increase with respect to $\tau$.

(ii) Consider the case of small sample sizes $(m, n \leq 10)$. For small values of $\tau(\tau \leq$ $0.25)$ the estimator $\underline{d}^{M L}$ has maximum percentage of relative risk performance and it is noticed near to $97.7 \%$, for moderate values of $\tau(0.75<\tau<2.0)$ the

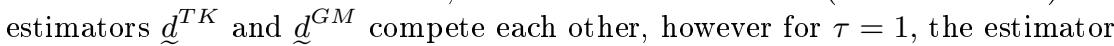
$\widetilde{d}^{G M}$ has the best performance. For large values of $\tau,(\tau>3)$ the estimator $\underline{d}^{B C 1}$ has the best performance.

Consider the case of moderate sample sizes $(12 \leq m, n \leq 20)$. For small values of $\tau(\tau<0.25)$ the estimator ${\underset{d}{d L}}^{M L}$ has the best performance, for moderate values 
of $\tau(0.75 \leq \tau<2.0)$, the estimator ${\underset{d}{d}}^{K S}$ and $\underline{d}^{G D}$ compete each other. For $\tau=1$ the estimator ${\underset{d}{d M}}^{G M}$ performs the best. For large values of $\tau$ the estimator $d^{B C 1}$ and $\underline{d}^{M L}$ compete each other.

Consider the case of large sample sizes $(m, n \geq 30)$. For small values of $\tau$ the

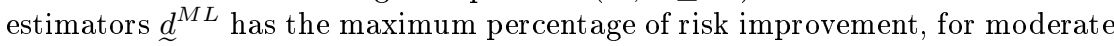

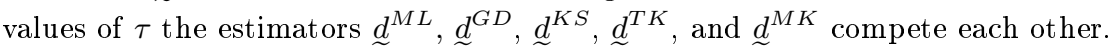
However for $\tau=1$ the estimator $\widetilde{d}^{G M}$ has the best performance. For large values

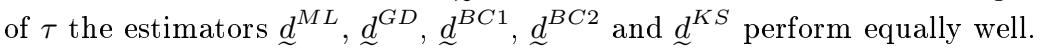

On the basis of the above discussion and observations the following recommendations may be done for the use of the estimators.

(i) We conclude from the above discussion that, none of the estimators completely dominate others in terms of the risk function for the full range of the parameters.

(ii) When the sample sizes are small that is $m, n \leq 10$, the estimators $d^{M L}$ and $d^{Y}$ can be used if $\tau$ is near to 0 . For values of $\tau$ in the neighborhood of 1 , the estimators ${\underset{d}{d K}}^{M K}$ and ${\underset{d}{d K}}^{T K}$ may be used, however for $\tau=1$, that is, when the variances are of the two populations are same, the estimator $\underline{d}^{G M}$ should be used. For large values of $\tau$ we recommend to use $\underline{d}^{B C 1}$.

(iii) When the sample sizes are from moderate to large the estimators ${\underset{\sim}{d L}}^{M L}{\underset{d}{d}}^{G D}$, or ${\underset{\sim}{d}}^{K S}$ may be used if $\tau$ is near to 0 , however for moderate values of $\tau$ we recommend to use either of the estimators ${\underset{d}{d}}^{G D},{\underset{\sim}{d S}}^{K S}{\underset{d}{d K}}^{d^{T K}}$, or ${\underset{\sim}{d}}^{M L}$. For values of $\tau=1$, the estimator $d^{G M}$ is strongly recommended to use. For large values of $\tau$, the estimators ${\underset{\sim}{d}}^{M L},{\underset{\sim}{d}}^{B C 1}$, or ${\underset{\sim}{d}}^{B C 2}$ may be used.

(iv) A similar type of observations have been made for other combinations of sample sizes and $\eta$. 
Table 1: Percentage of Relative Risk Improvements of Various Estimators of Normal Quantiles with $\eta=1.960,(m, n)=(8,8),(12,12),(20,20),(40,40)$

\begin{tabular}{|c|c|c|c|c|c|c|c|c|}
\hline$\tau \downarrow$ & $P R 1$ & $\overline{P R 2}$ & $P R 3$ & $P R 5$ & $P R 6$ & $P R 7$ & $P R 8$ & $P R 10$ \\
\hline \multirow{4}{*}{0.05} & 98.72 & 74.20 & 98.72 & 55.40 & 53.86 & 30.17 & 98.46 & 98.73 \\
\hline & 98.76 & 74.20 & 98.76 & 70.66 & 75.75 & 63.20 & 98.52 & 98.76 \\
\hline & 98.76 & 74.19 & 98.76 & 83.70 & 88.49 & 84.65 & 98.54 & 98.76 \\
\hline & 98.80 & 74.22 & 98.80 & 92.81 & 94.90 & 95.05 & 98.58 & 98.80 \\
\hline \multirow{4}{*}{0.15} & 89.50 & 68.21 & 89.45 & 50.46 & 48.60 & 27.28 & 88.06 & 89.50 \\
\hline & 89.73 & 68.29 & 89.74 & 64.02 & 68.62 & 57.31 & 88.45 & 89.76 \\
\hline & 89.75 & 68.39 & 89.79 & 76.20 & 80.40 & 76.96 & 88.59 & 89.79 \\
\hline & 90.16 & 68.66 & 90.20 & 84.69 & 86.62 & 86.77 & 89.05 & 90.20 \\
\hline \multirow{4}{*}{0.25} & 75.29 & 59.03 & 75.28 & 42.83 & 40.68 & 22.89 & 73.34 & 75.43 \\
\hline & 75.77 & 59.25 & 75.89 & 54.65 & 57.81 & 48.33 & 73.97 & 75.93 \\
\hline & 76.07 & 59.45 & 76.28 & 64.74 & 68.12 & 65.22 & 74.43 & 76.29 \\
\hline & 76.32 & 59.75 & 76.57 & 71.96 & 73.48 & 73.62 & 74.77 & 76.58 \\
\hline \multirow{4}{*}{0.50} & 40.53 & 36.89 & 41.99 & 24.76 & 22.66 & 12.60 & 41.28 & 41.92 \\
\hline & 41.25 & 37.75 & 43.22 & 31.97 & 33.03 & 27.52 & 42.35 & 43.21 \\
\hline & 41.60 & 38.23 & 44.02 & 37.94 & 39.44 & 37.74 & 43.01 & 44.03 \\
\hline & 41.79 & 38.54 & 44.60 & 42.16 & 42.88 & 42.95 & 43.48 & 44.60 \\
\hline \multirow{4}{*}{0.75} & 17.86 & 24.31 & 23.92 & 15.07 & 13.37 & 07.07 & 24.71 & 23.45 \\
\hline & 18.06 & 24.87 & 25.04 & 19.29 & 19.57 & 15.87 & 25.52 & 24.77 \\
\hline & 17.79 & 24.86 & 25.47 & 22.42 & 22.99 & 21.74 & 25.63 & 25.40 \\
\hline & 17.45 & 25.02 & 25.81 & 24.66 & 24.94 & 24.90 & 25.81 & 25.79 \\
\hline \multirow{4}{*}{1.00} & -0.86 & 15.68 & 13.55 & 09.61 & 08.38 & 04.16 & 15.01 & 12.70 \\
\hline & -0.18 & 16.58 & 15.10 & 12.37 & 12.39 & 09.65 & 16.14 & 14.64 \\
\hline & 01.15 & 17.20 & 16.38 & 14.65 & 14.93 & 13.72 & 16.98 & 16.24 \\
\hline & 00.56 & 16.99 & 16.51 & 15.83 & 15.97 & 15.76 & 16.85 & 16.48 \\
\hline \multirow{4}{*}{1.25} & -15.31 & 10.10 & 08.78 & 06.77 & 05.86 & 02.75 & 10.05 & 07.96 \\
\hline & -17.90 & 09.50 & 09.08 & 08.06 & 08.01 & 06.04 & 09.81 & 08.69 \\
\hline & -16.36 & 10.49 & 10.32 & 09.62 & 09.72 & 08.80 & 10.81 & 10.18 \\
\hline & -16.82 & 10.67 & 11.02 & 10.71 & 10.78 & 10.60 & 11.17 & 10.97 \\
\hline \multirow{4}{*}{1.50} & -31.46 & 04.87 & 05.88 & 05.07 & 04.33 & 01.97 & 06.51 & 05.19 \\
\hline & -32.18 & 04.77 & 06.44 & 06.00 & 05.87 & 04.38 & 06.62 & 06.29 \\
\hline & -34.66 & 04.40 & 06.96 & 06.76 & 06.80 & 06.21 & 06.68 & 06.88 \\
\hline & -34.23 & 05.07 & 08.09 & 07.90 & 07.92 & 07.80 & 07.54 & 08.08 \\
\hline \multirow{4}{*}{2.00} & -67.82 & $\begin{array}{l}-5.69 \\
\end{array}$ & 02.45 & 02.94 & 02.54 & 01.13 & 01.45 & 02.30 \\
\hline & -70.73 & -6.03 & 03.48 & 03.54 & 03.47 & 02.61 & 01.95 & 03.46 \\
\hline & -69.55 & -4.90 & 04.53 & 04.35 & 04.37 & 03.99 & 03.00 & 04.51 \\
\hline & -72.31 & -5.85 & 04.57 & 04.52 & 04.53 & 04.48 & 02.63 & 04.57 \\
\hline \multirow{4}{*}{2.50} & -116.05 & -18.14 & 01.11 & 01.89 & 01.68 & 00.77 & -1.87 & 01.24 \\
\hline & -115.39 & -17.45 & 02.27 & 02.46 & 02.40 & 01.82 & -0.84 & 02.38 \\
\hline & -120.07 & -18.71 & 02.59 & 02.68 & 02.66 & 02.47 & -1.00 & 02.68 \\
\hline & -119.29 & -18.49 & 02.74 & 02.78 & 02.77 & 02.75 & -0.81 & 02.76 \\
\hline \multirow{4}{*}{3.00} & -169.15 & -31.75 & 00.49 & 01.45 & 01.21 & 00.57 & -4.27 & 01.09 \\
\hline & -170.01 & -31.47 & 01.27 & 01.56 & 01.57 & 01.22 & -3.49 & 01.42 \\
\hline & -172.46 & -31.42 & 02.11 & 02.12 & 02.09 & 01.93 & -2.55 & 02.17 \\
\hline & -176.39 & -32.49 & 02.20 & 02.17 & 02.17 & 02.14 & -2.59 & 02.20 \\
\hline \multirow{4}{*}{4.00} & -293.23 & -61.96 & 00.38 & 00.88 & 00.78 & 00.36 & -6.55 & 00.80 \\
\hline & -304.09 & -65.67 & 00.48 & 00.81 & 00.82 & 00.66 & -7.06 & 00.68 \\
\hline & -311.09 & -66.74 & 01.19 & 01.20 & 01.19 & 01.10 & -6.02 & 01.23 \\
\hline & -319.53 & -69.42 & 01.03 & 01.06 & 01.06 & 01.05 & -6.51 & 01.05 \\
\hline
\end{tabular}


Table 2: Percentage of Relative Risk Improvements of Various Estimators of Normal Quantiles with $\eta=1.960,(m, n)=(4,10),(12,20),(30,40)$

\begin{tabular}{|c|c|c|c|c|c|c|c|c|c|}
\hline$\tau$ & $\overline{P R 1}$ & $P R 2$ & $P R 3$ & $P R 4$ & $P R 5$ & $P R 6$ & $P R 8$ & $P R 9$ & $P R 10$ \\
\hline \multirow{3}{*}{0.05} & $\begin{array}{l}98.74 \\
\end{array}$ & 90.72 & 98.72 & 98.74 & 48.01 & 53.65 & 98.51 & 98.53 & 98.74 \\
\hline & 98.69 & 84.86 & 98.69 & 98.69 & 79.81 & 83.67 & 98.53 & 98.54 & 98.69 \\
\hline & 98.67 & 80.62 & 98.67 & 98.67 & 91.94 & 93.71 & 98.50 & 98.50 & 98.67 \\
\hline \multirow{3}{*}{0.15} & 89.99 & 82.96 & 89.77 & 89.92 & 43.60 & 48.41 & 88.72 & 88.83 & 89.98 \\
\hline & 89.34 & 77.33 & 89.35 & 89.35 & 72.34 & 75.63 & 88.45 & 88.46 & 89.36 \\
\hline & 89.21 & 73.63 & 89.24 & 89.24 & 83.16 & 84.76 & 88.31 & 88.31 & 89.24 \\
\hline \multirow{3}{*}{0.25} & 76.50 & 71.03 & 75.95 & 76.33 & 36.96 & 40.55 & 74.39 & 74.57 & 76.48 \\
\hline & 75.22 & 66.08 & 75.27 & 75.28 & 61.08 & 63.67 & 73.81 & 73.84 & 75.30 \\
\hline & 74.94 & 62.96 & 75.07 & 75.07 & 69.93 & 71.23 & 73.52 & 73.53 & 75.08 \\
\hline \multirow{3}{*}{0.50} & 46.57 & 44.83 & 45.59 & 46.46 & 22.71 & 23.98 & 44.43 & 44.65 & 46.53 \\
\hline & 43.61 & 41.04 & 44.23 & 44.28 & 36.01 & 37.19 & 42.89 & 42.93 & 44.31 \\
\hline & 42.16 & 39.03 & 43.48 & 43.49 & 40.56 & 41.16 & 42.17 & 42.17 & 43.50 \\
\hline \multirow{3}{*}{0.75} & 29.00 & 29.96 & 28.53 & 29.48 & 14.74 & 15.03 & 28.38 & 28.58 & 28.88 \\
\hline & 24.71 & 27.02 & 27.10 & 27.17 & 22.40 & 22.85 & 26.75 & 26.77 & 27.11 \\
\hline & 20.91 & 25.07 & 25.53 & 25.53 & 24.10 & 24.37 & 25.31 & 25.32 & 25.52 \\
\hline \multirow{3}{*}{1.00} & 18.38 & 21.72 & 19.56 & 20.22 & 10.59 & 10.54 & 20.24 & 20.38 & 19.29 \\
\hline & 11.35 & 18.16 & 17.45 & 17.48 & 14.86 & 15.01 & 17.78 & 17.79 & 17.36 \\
\hline & 07.2 & 17.09 & 16.77 & 16.77 & 15.93 & 16.05 & 16.96 & 16.96 & 16.74 \\
\hline \multirow{3}{*}{1.25} & 09.62 & 15.57 & 13.89 & 14.02 & 07.97 & 07.77 & 15.03 & 15.12 & 13.32 \\
\hline & 02.77 & 13.30 & 12.91 & 12.93 & 11.06 & 11.09 & 13.50 & 13.51 & 12.74 \\
\hline & -4.45 & 11.25 & 11.45 & 11.45 & 11.02 & 11.02 & 11.77 & 11.77 & 11.41 \\
\hline \multirow{3}{*}{1.50} & 03.39 & 1.56 & 10.57 & 10.33 & 06.29 & 06.10 & 12.03 & 12.07 & 09.84 \\
\hline & -8.37 & 07.64 & 09.18 & 09.15 & 08.16 & 08.13 & 09.66 & 09.65 & 08.99 \\
\hline & -15.42 & 06.25 & 08.30 & 08.29 & 07.98 & 07.99 & 08.34 & 08.33 & 08.27 \\
\hline \multirow{3}{*}{2.00} & -13.23 & 01.65 & 05.59 & 03.91 & 04.05 & 03.82 & 06.86 & 06.78 & 04.87 \\
\hline & -29.23 & -1.70 & 05.12 & 05.03 & 04.86 & 04.83 & 05.06 & 05.03 & 05.00 \\
\hline & -44.68 & -4.36 & 04.66 & 04.64 & 04.65 & 04.64 & 03.58 & 03.57 & 04.65 \\
\hline \multirow{3}{*}{2.50} & -29.19 & -6.74 & 03.43 & 00.82 & 02.96 & 02.77 & 04.27 & 04.11 & 02.86 \\
\hline & -54.92 & 2.23 & 03.36 & 03.25 & 03.32 & 03.29 & 02.20 & 02.15 & 03.32 \\
\hline & -73.59 & -14.60 & 02.94 & 02.93 & 02.98 & 02.97 & 00.74 & 00.73 & 02.95 \\
\hline \multirow{3}{*}{3.00} & -49.24 & -17.00 & 02.39 & -0.89 & 02.31 & 02.15 & 02.43 & 02.18 & 01.92 \\
\hline & -87.01 & -25.22 & 02.26 & 02.13 & 02.37 & 02.34 & -0.28 & -0.36 & 02.31 \\
\hline & -110.99 & -26.79 & 02.34 & 02.33 & 02.34 & 02.31 & -0.94 & -0.96 & 02.36 \\
\hline \multirow{3}{*}{3.50} & -76.25 & -31.59 & 00.89 & -3.42 & 01.63 & 01.51 & -0.37 & -0.76 & 00.76 \\
\hline & -120.65 & -38.15 & 01.77 & 01.66 & 01.85 & 01.83 & -1.67 & -1.76 & 01.82 \\
\hline & -159.48 & -43.07 & 01.61 & 01.59 & 01.63 & 01.64 & -3.08 & -3.10 & 01.62 \\
\hline \multirow{3}{*}{3.75} & -83.63 & -34.56 & 01.41 & -2.41 & 01.64 & 01.53 & 00.17 & -0.21 & 01.22 \\
\hline & -138.71 & -45.64 & 01.33 & 01.21 & 01.49 & 01.47 & -2.67 & -2.77 & 01.41 \\
\hline & -181.46 & -49.60 & 01.60 & 01.59 & 01.59 & 01.58 & -3.22 & -3.25 & 01.61 \\
\hline \multirow{3}{*}{4.00} & -99.21 & -42.8 & 00.90 & -3.31 & 01.42 & 01.31 & -0.97 & -1.42 & 00.81 \\
\hline & -157.38 & -53.73 & 00.97 & 00.86 & 01.18 & 01.16 & -3.7 & -3.80 & 01.08 \\
\hline & -199.50 & -55.59 & 01.39 & 01.39 & 01.38 & 01.37 & -3.54 & -3.57 & 01.40 \\
\hline
\end{tabular}


Table 3: Percentage of Relative Risk Improvements of Various Estimators of Normal Quantiles with $\eta=1.960,(m, n)=(10,4),(20,12),(40,30)$

\begin{tabular}{|c|c|c|c|c|c|c|c|c|c|}
\hline$\tau$ & $P R 1$ & $P R 2$ & $P R 3$ & $P R 4$ & $P R 5$ & $P R 6$ & $P R 8$ & PR9 & PR10 \\
\hline \multirow{3}{*}{0.05} & 96.66 & 47.61 & 96.65 & 96.61 & 31.74 & 06.15 & 96.10 & 96.04 & 96.66 \\
\hline & 97.65 & 59.62 & 97.65 & 97.65 & 73.60 & 76.84 & 97.28 & 97.28 & 97.65 \\
\hline & 98.16 & 66.31 & 98.16 & 98.16 & 90.21 & 93.39 & 97.90 & 97.89 & 98.16 \\
\hline \multirow{3}{*}{0.15} & 74.93 & 38.57 & 74.89 & 73.82 & 25.03 & 04.75 & 73.28 & 73.07 & 75.01 \\
\hline & 81.95 & 51.36 & 82.00 & 81.98 & 62.19 & 64.41 & 80.44 & 80.41 & 82.01 \\
\hline & 85.51 & 58.82 & 85.58 & 85.57 & 78.61 & 81.31 & 84.22 & 84.21 & 85.58 \\
\hline \multirow{3}{*}{0.25} & 51.02 & 28.76 & 51.30 & 49.01 & 17.79 & 03.27 & 50.24 & 50.00 & 51.33 \\
\hline & 60.73 & 40.31 & 61.25 & 61.21 & 46.87 & 48.12 & 59.58 & 59.54 & 61.27 \\
\hline & 66.65 & 47.91 & 67.08 & 67.08 & 61.81 & 63.78 & 65.37 & 65.36 & 67.09 \\
\hline \multirow{3}{*}{0.50} & 11.92 & 13.43 & 17.02 & 15.51 & 07.06 & 01.24 & 18.18 & 18.11 & 16.37 \\
\hline & 23.63 & 21.38 & 27.08 & 26.99 & 21.28 & 21.32 & 26.88 & 26.85 & 27.00 \\
\hline & 30.09 & 27.26 & 33.27 & 33.26 & 30.78 & 31.58 & 32.56 & 32.55 & 33.27 \\
\hline \multirow{3}{*}{0.75} & -7.17 & 07.28 & 05.65 & 05.53 & 03.32 & 00.57 & 07.09 & 07.16 & 04.80 \\
\hline & 01.51 & 12.33 & 12.52 & 12.51 & 10.49 & 10.37 & 13.11 & 13.11 & 12.31 \\
\hline & 07.33 & 16.57 & 17.34 & 17.34 & 16.42 & 16.67 & 17.52 & 17.52 & 17.31 \\
\hline \multirow{3}{*}{1.00} & -22.64 & 04.15 & 01.39 & 02.30 & 01.87 & 00.34 & 02.23 & 02.41 & 01.16 \\
\hline & -13.11 & 07.75 & 06.84 & 06.88 & 06.14 & 05.98 & 07.26 & 07.27 & 06.68 \\
\hline & -9.14 & 10.03 & 09.55 & 09.55 & 09.27 & 09.34 & 09.76 & 09.77 & 09.52 \\
\hline \multirow{3}{*}{1.25} & -37.14 & 01.91 & -0.45 & 00.84 & 01.11 & 00.21 & -0.44 & -0.18 & -0.40 \\
\hline & -27.24 & 04.17 & 04.00 & 04.06 & 03.82 & 03.70 & 03.75 & 03.78 & 03.96 \\
\hline & -21.59 & 06.40 & 06.57 & 06.58 & 06.36 & 06.38 & 06.46 & 06.46 & 06.55 \\
\hline \multirow{3}{*}{1.50} & -50.31 & 00.63 & -0.75 & 00.55 & 00.87 & 00.17 & -1.31 & -1.01 & -0.17 \\
\hline & -42.87 & 00.85 & 02.20 & 02.30 & 02.41 & 02.32 & 01.18 & 01.23 & 02.27 \\
\hline & -38.20 & 02.26 & 04.45 & 04.46 & 04.37 & 04.39 & 03.55 & 03.56 & 04.45 \\
\hline \multirow{3}{*}{2.00} & -89.75 & -3.66 & -2.23 & -0.43 & 00.41 & 00.09 & -4.51 & -4.06 & -0.24 \\
\hline & -77.46 & -4.60 & 01.17 & 01.26 & 01.41 & 01.33 & -1.18 & -1.11 & 01.33 \\
\hline & -72.94 & -5.01 & 02.44 & 02.45 & 02.46 & 02.45 & 00.30 & 00.31 & 02.46 \\
\hline \multirow{3}{*}{2.50} & -130.42 & -7.14 & $\begin{array}{l}-2.33 \\
\end{array}$ & -0.55 & 00.26 & 00.06 & -5.74 & -5.21 & -0.18 \\
\hline & -125.44 & -11.86 & 00.60 & 00.68 & 00.85 & 00.81 & -3.25 & -3.16 & 00.78 \\
\hline & -115.91 & -13.22 & 1.64 & 01.65 & 01.64 & 01.63 & -1.63 & -1.61 & 01.66 \\
\hline \multirow{3}{*}{3.00} & -190.10 & -12.31 & -2.55 & -0.66 & 00.13 & 00.03 & -7.50 & -6.85 & -0.24 \\
\hline & -175.49 & -19.09 & 00.36 & 00.42 & 00.56 & 00.53 & -4.33 & -4.23 & 00.50 \\
\hline & -162.38 & -21.35 & 01.32 & 01.32 & 01.29 & 01.28 & -2.49 & -2.47 & 01.32 \\
\hline \multirow{3}{*}{3.50} & -250.22 & -17.03 & -2.10 & -0.49 & 00.10 & 00.03 & -8.03 & -7.31 & -0.17 \\
\hline & -234.56 & -27.38 & 00.29 & 00.34 & 00.43 & 00.42 & -5.11 & -5.00 & 00.40 \\
\hline & -225.80 & -34.25 & 00.75 & 00.76 & 00.78 & 00.77 & -4.54 & -4.51 & 00.78 \\
\hline \multirow{3}{*}{3.75} & -285.75 & -19.42 & -1.88 & -0.42 & 00.16 & 00.04 & -7.97 & -7.23 & 00.06 \\
\hline & -264.67 & -31.69 & 00.20 & 00.25 & 00.36 & 00.33 & -5.41 & -5.30 & 00.32 \\
\hline & -264.47 & -41.68 & 00.55 & 00.56 & 00.59 & 00.60 & -5.42 & -5.39 & 00.57 \\
\hline \multirow{3}{*}{4.00} & -328.00 & -23.98 & -2.36 & -0.77 & 00.08 & 00.02 & -9.20 & -8.41 & -0.09 \\
\hline & -296.86 & -35.41 & 00.37 & 00.40 & 00.44 & 00.40 & -5.16 & -5.04 & 00.45 \\
\hline & -296.24 & -47.50 & 00.50 & 00.51 & 00.53 & 00.53 & -5.68 & -5.65 & 00.52 \\
\hline
\end{tabular}

\section{Concluding Remarks and Illustrative Examples}

We note here that, in the literature most of the results on estimation of quantiles are for a single parameter $\theta=\mu+\eta \sigma$ either using one or more populations. In this article, we consider the simultaneous estimation of the quantile vector $\underset{\sim}{\theta}=\left(\theta_{1}, \theta_{2}\right)$ which is important from an application point of view. The loss function is taken as the sum of the quadratic loss functions. It should be noted that, Kumar and Tripathy [9] considered this model and estimated the first component $\theta_{1}$ with respect 

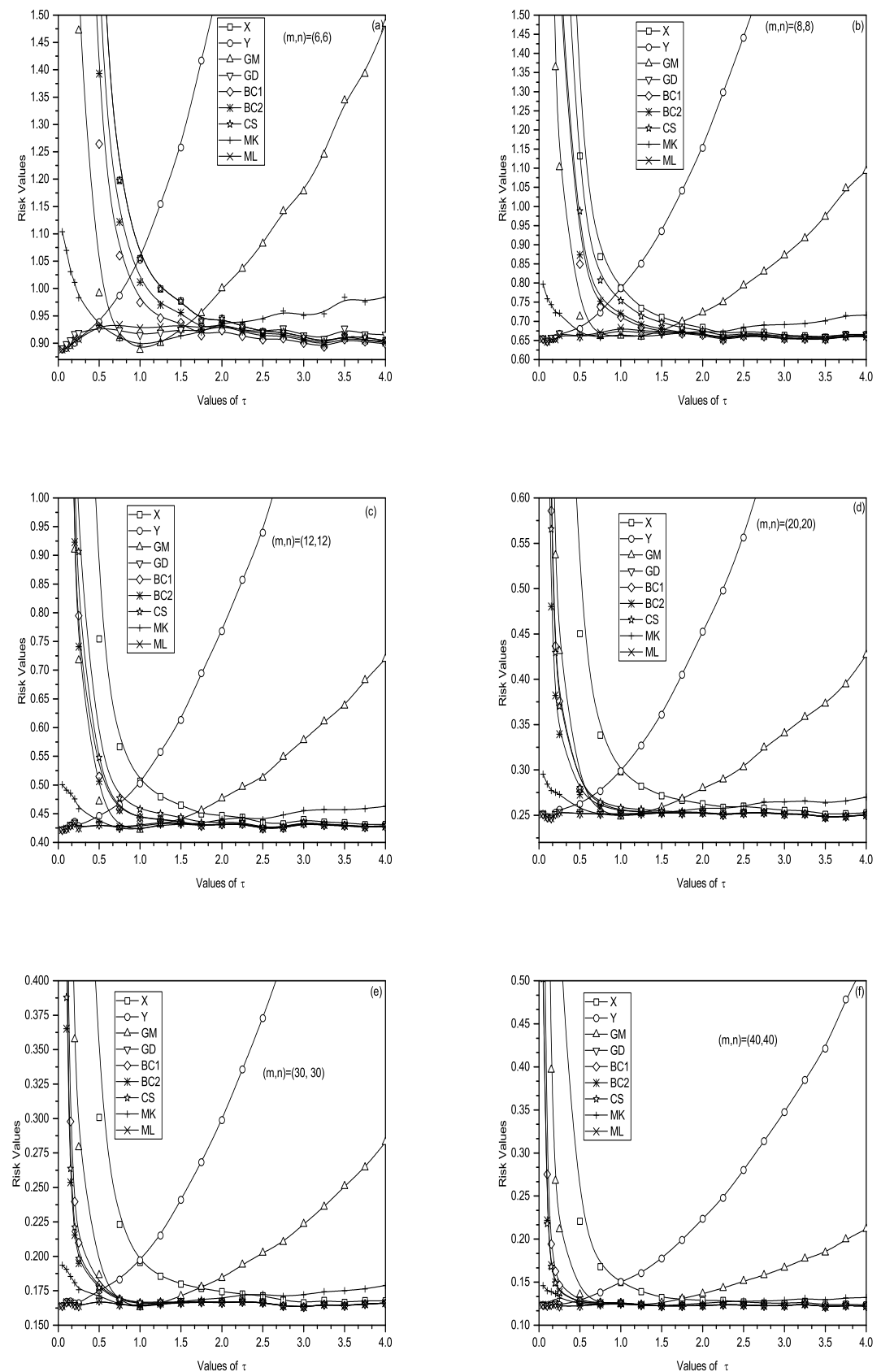

Figure 1. Comparison of risk values of various estimators for quantile vector $\left(\theta_{1}, \theta_{2}\right)$ when $\eta=1.960$ and the sample sizes are equal 

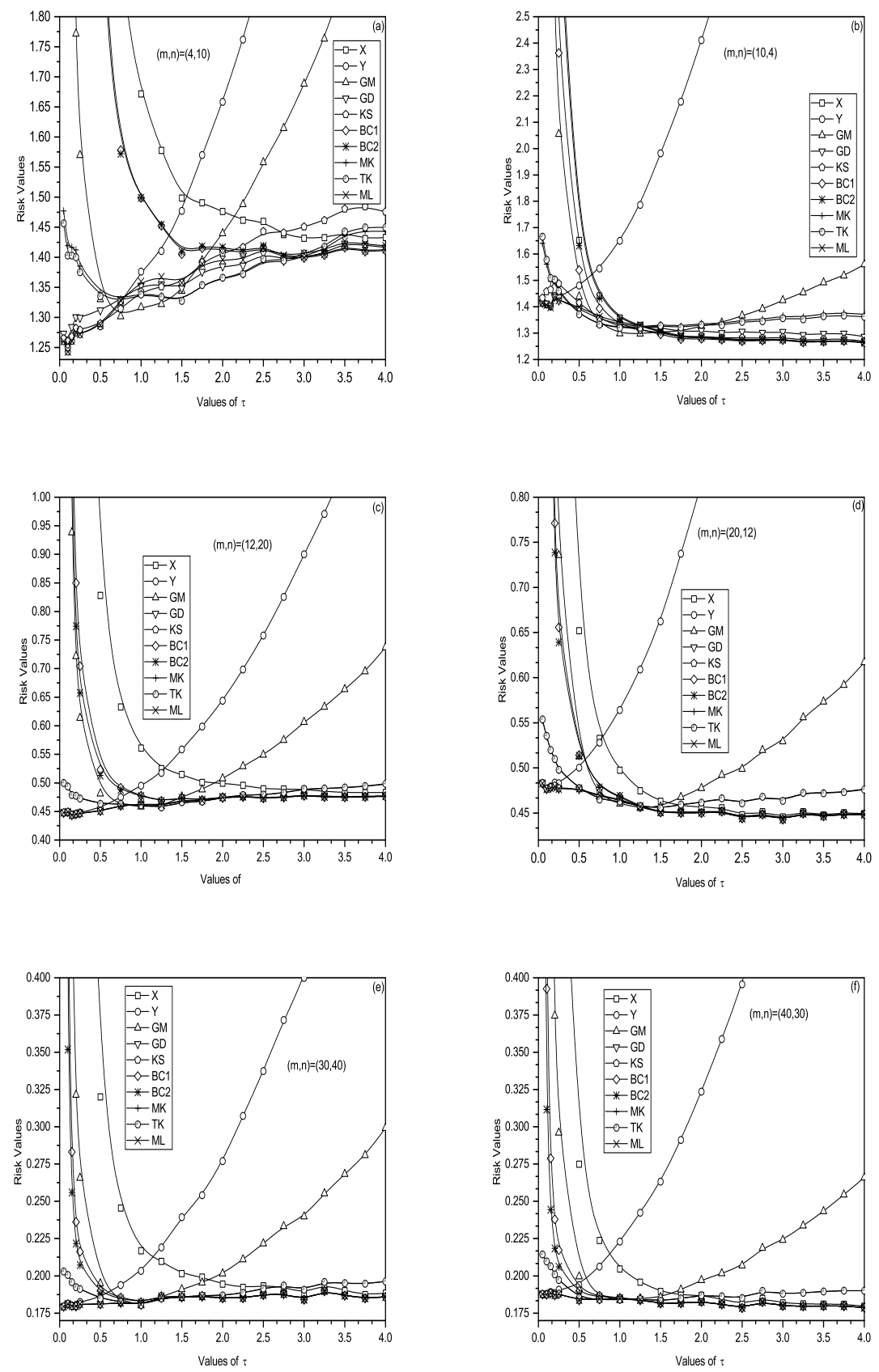

Figure 2. Comparison of risk values of various estimators for quantile vector $\left(\theta_{1}, \theta_{2}\right)$ when $\eta=1.960$ and the sample sizes are unequal 
to a quadratic loss function. We have implemented the Brewster and Zidek [3] technique to the case of estimating a vector parameter, which is interesting. Further we have proposed some new estimators such as the $d^{Y}, d^{G M}$, and $d^{M L}$ which was not considered by them. First, we derived sufficient conditions for improving equivariant estimators and in the process some complete class results obtained. We have constructed some improved estimators using one of our result obtained in Section 2. However, the analytical comparison of these estimators is not possible. We have conducted a detailed simulation study to numerically compare these estimators which can be used in practice. Our conclusions regarding the use of the estimators are completely based on the simulation study as no analytical comparison is possible among all the estimators. It will be interesting to generalize the results to the case of $k \geq 3$ normal populations, where proving inadmissibility of these estimators will be challenging. Below we present some examples where our model fits well and also compute the estimates for practical purposes. In the examples below we have taken the value of $\eta=1.960$ for convenient.

5.1. Example. We consider the example discussed in Hines et al. [6], (p. 290). Suppose a manufacturer of video display units produces two micro circuit designs design $\mathrm{A}$ and design $\mathrm{B}$. He wants to test whether the two design produce same current flow. The summarized data for design $\mathrm{A}$ are given by $m=15, \bar{x}=24.2$, $s_{1}^{2}=10$ where as the data for design B are given by $n=10, \bar{y}=23.9, s_{2}^{2}=20$. It is also given that both the data follow normal distributions with a common mean. The experimental conditions ensures that the variances are unequal. This is a situation where our model will be very much useful. The several estimators for quantiles are calculated as $\underline{d}^{X}=(25.97,26.71), \underline{d}^{Y}=(25.67,26.41), \underline{d}^{G M}=$ $(25.85,26.59), d^{G D}=(25.92,26.65), d^{K S}=(25.92,26.66), d^{B C 1}=(25.97,26.71)$, $\stackrel{d}{d}^{B C 2}=(25.94,26.68), \stackrel{d}{d}^{M K}=(25.88,26.61), \stackrel{\sim}{d T K}^{T K}=(25.88,26.61)$ and $\stackrel{\sim}{d}^{M L}=$ $(25.92,26.65)$. If the variances of both the data set differ significantly we may use either the estimator $d^{G D}, d^{M L}$, or $\underline{d}^{B C 1}$. If the variances differ marginally we may use either $\underline{d}^{K S}$, or $d^{M K}$.

5.2. Example. Rohatgi and Saleh [13], (p.515) discussed one example regarding the mean life time (in hours) of light bulbs. Suppose a random sample of 9 bulbs has sample mean 1309 hours with standard deviation of 420 hours. A second sample of 16 bulbs chosen from a different batch has sample mean 1205 hours and standard deviation 390 hours. A two sample t-test fails to reject the hypothesis that the means are equal. This is a situation where our model will be useful. Suppose we want to know the life time of both the bulbs at any instant of time then we can use our estimators. The various estimators are calculated as $d^{X}=(1543.45,1526.70), d^{Y}=(1439.45,1422.70), d^{G M}=(1476.89,1460.14)$, $\widetilde{d}^{G \widetilde{D}}=(1460.82,1444.08), \underline{d}^{K S}=(1458.47,1441.73), \widetilde{d}^{B C 1}=(1501.44,1484.69)$, $d^{B C 2}=(1498.38,1481.64), d^{M K}=(1474.51,1457.77), d^{T K}=(1474.18,1457.43)$ and ${\underbrace{M L}}^{M L}(1457.08,1440.33)$. Also a F-test fails to reject the hypothesis that the population variances are equal. In this situation we recommend to use either ${\underset{\sim}{d K}}^{T K}$, or $\stackrel{\sim}{d}^{M K}$. 


\section{References}

[1] Alexander, T. L. and Chandrasekar, B. Simultaneous equivariant estimation of the parameters of matrix scale and matrix location-scale models, Statistical Papers 46, 483-507, 2005.

[2] Bai, S. K. and Durairajan, T. M. Simultaneous equivariant estimation of the parameters of linear models, Statistical Papers 39, 125-134, 1998.

[3] Brewster, J. F. and Zidek, J. V. Improving on equivariant estimators, Annals of Statistics 2, 21-38, 1974.

[4] Brown, L. D. and Cohen, A. Point and confidence estimation of a common mean and recovery of interblock information, Annals of Statistics 2 (5), 963-976, 1974.

[5] Chang, C. H. and Pal, N. Testing on the common mean of several normal distributions, Computational Statistics and Data Analysis 53, 321-333, 2008.

[6] Hines, W. W., Montgomery, D. C., Goldsman, D. M. and Borror, D. M. Probability and Statistics in Engineering, John Wiley, New Work, 2008.

[7] Keating, J. P. and Tripathi, R. C. Percentiles, estimation of 'Encyclopedia of Statistical Sciences, VI, 668-674, 1985.

[8] Kiefer, J. Invariance minimax sequential estimation and contineous time processes, Annals of Mathematical Statistics 28, 573-601, 1957.

[9] Kumar, S. and Tripathy, M. R. Estimating quantiles of normal populations with a common mean, Communications in Statistics-Theory and Methods 26, 115-118, 2011.

[10] Lin, S. H. and Lee, J. C. Generalized inferences on the common mean of several normal populations, Journal of Statistical Planning and Inferences 134, 568-582, 2005.

[11] Moore, B. and Krishnamoorthy, K. Combining independent normal sample means by weighting with their standard errors, Journal of Statistical Computation and Simulations 58, 145$153,1997$.

[12] Pal, N., Lin, J. J., Chang, C. H. and Kumar, S. A revisit to the common mean problem: Comparing the maximum likelihood estimator with the Graybill-Deal estimator, Computational Statistics and Data Analysis 51, 5673-5681, 2007.

[13] Rohatgi, V. K. and Saleh, A. Md. E. An Introduction to Probability and Statistics, John Wiley, 2nd Edition, New Work, 2003.

[14] Rukhin, A. L. A class of minimax estimators of a normal quantile, Statistics and Probability Letters 1, 217-221, 1983.

[15] Rukhin, A. L. Admissibility and minimaxity results in the estimation problem of exponential quantiles, Annals of Statistics 14, 220-237, 1986.

[16] Saleh, A. K. Md. E. Estimating quantiles of exponential distributions, In:Statistics and Related Topics, Csorgo, M., Dawson, D., Rao, J. N. K., Saleh, A. K. Md. E. (Eds.), North Holland, Amsterdam, 279-283, 1981.

[17] Sharma, D. and Kumar, S. Estimating quantiles of exponential populations, Statistics and Decisions, 12, 343-352, 1994.

[18] Tripathy, M. R. and Kumar, S. Estimating a common mean of two normal populations, Journal of Statistical Theory and Applications 9 (2), 197-215, 2010.

[19] Tsukuma, T. Simultaneous estimation of restricted location parameters based on permutation and sign-change, Statistical Papers, 53, 915-934, 2012.

[20] Vazquez, G., Duval, S., Jacobs Jr, D. R. and Silventoinen, K. Comparison of body mass index, waist cicumference and waist/hip ratio in predicting incident diabetes: A meta analysis, Epidemilogic Reviews 29 (1), 115-128, 2007.

[21] Zidek, J. V. Inadmissibility of the best invariant estimators of extreme quantiles of the normal law under squared error loss, Annals of Mathematical Statistics 40 (5), 1801-1808, 1969.

[22] Zidek, J. V. Inadmissibility of a class of estimators of a normal quantile, The Annals of Mathematical Statistics 42 (4), 1444-1447, 1971. 